\title{
Tangence
}

\section{L'inscription corporelle de la vitesse : la « nouvelle décadence » dans Crash de J. G. Ballard}

\section{Donald Bruce}

Numéro 55, septembre 1997

La vitesse

URI : https://id.erudit.org/iderudit/025951ar

DOI : https://doi.org/10.7202/025951ar

Aller au sommaire du numéro

Éditeur(s)

Tangence

ISSN

0226-9554 (imprimé)

1710-0305 (numérique)

Découvrir la revue

Citer cet article

Bruce, D. (1997). L'inscription corporelle de la vitesse : la «nouvelle décadence " dans Crash de J. G. Ballard. Tangence, (55), 118-137.

https://doi.org/10.7202/025951ar d'utilisation que vous pouvez consulter en ligne.

https://apropos.erudit.org/fr/usagers/politique-dutilisation/ 


\section{L'inscription corporelle de la vitesse: la «nouvelle décadence» dans Crash de J. G. Ballard}

Donald Bruce

...I've always tried to follow accurately, like a cruise missile, the contours of the period in which I'm living.

James Ballard ${ }^{1}$

This author is beyond psychiatric help. Do not publish!

- un lecteur chez l'éditeur anglais de J. G. Ballard, après voir lu Crash ${ }^{2}$

Depuis déjà longtemps l'homme habite un environnement qui est de plus en plus sa propre création. Pourtant, depuis à peu près deux siècles cet environnement produit des objets fétichisés dans une nouvelle configuration du temps et de l'espace, où la frontière entre l'homme et la machine parfois s'estompe ${ }^{3}$. Nombreux sont les textes de la fin du siècle passé où les auteurs explorent (consciemment ou inconsciemment) la manière dont l'homme et le monde technologisé s'interpénètrent. Il y a un siècle, une des réponses à ce nouveau rapport entre l'homme et un environnement en train de muer radicalement se voyait dans la décadence, l'expression esthétique d'un phénomène sociohistorique qui s'étendait partout en Europe, mais dont la version française était particulièrement intense. Relevant paradoxalement à la fois de l'avant-garde et de la réaction, cette notion de décadence se range pourtant dans un courant discursif encore plus

1 David Pringle, "Interview with J. G. Ballard", J. G. Ballard. A Primary and Secondary Bibliography, Boston, G. K. Hall, 1984, p.15.

2 "Crash: About the Production", URL: http//:www.flf.com/crash/allnotes.htm (7 janvier 1997).

3 Voir les analyses de Jacques Noiray, Le romancier et la macbine. L'image de la machine dans le roman français (1850-1900), 2 vol. Paris, José Corti, $1981 / 1982$. 
vaste, celui de dégénérescence, qui va s'intensifiant vers la fin du siècle, surtout depuis la guerre franco-prussienne de 1870, la chute du Second Empire, et la Commune.

De nombreux éléments discursifs de ce qu'on appelle la décadence se retrouvent à l'époque actuelle dans les réponses que formule le vingtième siècle finissant aux questions posées par l'existence de "l'homme technologisé". Dans sa représentation du banal quotidien et de l'expérience physique extrême, en y mêlant l'hyper-réalisme avec la fantaisie visionnaire, le roman Crash $^{1}$ de James Ballard cherche à décrire un état d'être qui correspond à la conscience et à la perception du monde technologisé de notre propre fin de siècle. Je me propose ici d'examiner quelques-unes des ressemblances et des différences entre les représentations de la décadence de la fin du siècle passé et leurs corollaires à l'époque actuelle. Car, à plusieurs points de vue le rapport entre le psychopathologique et le sociopathologique se présente dans les deux cas de manière semblable, mais non identique. Chacun à sa façon, $\grave{A}$ rebours (1884) de J.-K. Huysmans ${ }^{5}$ et Crash (1973) de James G. Ballard ${ }^{6}$, incorporent des éléments significatifs du discours "décadent". Les deux textes ont même acquis des réputations tout à fait particulières: le premier en est venu à servir de synonyme à la "décadence fin de siècle" et signale tout un genre; le second, en partie grâce à la récente version filmique de David Cronenberg $(1995)^{7}$, a perdu son statut de "roman de culte" pour trouver un public plus large vingt ans après sa publication, choque une autre fin de siècle par ses hardiesses inattendues, et semble renvoyer à une pathologie contemporaine toujours mal comprise. Comme nous verrons dans la seconde partie de ce texte, un des principaux éléments qui sert à différencier la nouvelle de l'ancienne décadence est la notion de vitesse: aux niveaux thématique et actantiel la vitesse devient un des catalyseurs technologiques de la (post-)modernité. Bien sûr, de nombreux autres éléments y jouent des rôles également déci-

4 l.es citations seront tirées de la traduction française de Robert Louit, Calmann-Lévy, coll. "SF Press Pocket ", 1974, et seront suivies du numéro de la page entre parenthèses.

5 J.-K. Huysmans, À rebours, Paris, Gallimard, coll. "Folio ", 1977.

6 James G. Ballard, Crash, London, Vintage, 1995. Pour éviter toute confusion possible, clans le texte, les références à "Ballard" renvoient au personnage; I"auteur" sera réservé à l'écrivain.

7 David Cronenberg, Crasb, London, Faber \& Faber, 1996. 
sifs, mais dans ce roman la vitesse possède un rôle clé. Mais "la vitesse" en quel sens? Il s'agit dans un premier temps de comprendre la vitesse au sens le plus littéral: la rapidité du mouvement des objets matériels dans le monde physique. Mais, ce sens est "vite" clépassé par d'autres sens : le mouvement rapide de l'information, la réaction de la société humaine aux changements de rythme imposés par les nouvelles technologies, et les interactions entre l'humain et le technologique grâce aux médiations de la vitesse. Dans un premier temps, il faudra préciser l'arrière-fond socio-discursil de la fin du siècle précédent pour comprendre la "nouvelle décadence" de Crash.

\section{La dégénérescence/décadence au $x{ }^{e}$ siècle}

C'est une "coïncidence" remarquable que le Petit Robert signale l'année 1857 pour le premier emploi du terme "dégénérescence" dans son sens médical: " $3^{e}$ (1857) Méd. Modification pathologique (d'un tissu, d'un organe) avec perturbations de leurs fonctions. Dégénérescence calcaire, colloïde, graisseuse, pigmentaire. Dégénérescence du tissu nerveux.

Cette date est particulièrement significative pour notre perspective car c'est le moment de la formation d'un noud discursif, un point d'intersection où des discours d'origine et d'intention asséz différentes reprennent des éléments thématiques et narratifs, ainsi que des images et des topoï communs tirés de l'archive clu discours social pour les insérer dans des projets discursifs particuliers. On reconnaîtra bien sûr l'année de la parution des Fleurs du mal de Charles Baudelaire et de Madame Bovary de Gustave Flaubert, événements qui occasionnent un remueménage juridique significatif autour de leur prétendue immoralité (lire dégénérescence). Ces textes ouvertement transgressifs à plusieurs niveaux semblent confirmer dans le domaine de l'esthétique ce que tant de bourgeois à l'époque redoutent depuis déjà longtemps: la civilisation moderne, et surtout celle de France, est en pleine chute morale, voire physique, les deux se confondant facilement aux yeux de la classe dominante. À la fois symptôme et caluse de la dégénérescence, ces textes semblent signaler clairement la maladie inguérissable qui ronge le pays depuis au moins la Révolution de 1789.

Est publié en même temps un troisième livre, moins bien connu de nos jours que les deux autres, mais d'une grande 
importance quand même pour la formation du discours sur la dégénérescence: il s'agit du traité médical du Docteur Bénédicte Morel, le Traité des dégénérescences pbysiques, intellectuelles et morales de l'espèce bumaine. Bien que le sujet ostensible du livre soit le crétinisme, cette maladie particulière n'est en fin de compte qu'une manifestation d'un mal encore plus vaste et généralisé qui est profondément enraciné dans la société contemporaine, celle de dégénérescence héréditaire. Le livre de Morel deviendra une sorte de texte "classique" pour les discours sociomédicaux des années suivantes, car il prolonge et codifie une réflexion déjà bien engagée sur la dégénérescence historique des sociétés européennes provoquée par ce qu'on croit être l'affaiblissement rapide du stock génétique et moral. Ce noyau discursif qui semble incorporer un nouveau "Savoir" sur l'homme réunit cles éléments tirés de sources variées, celles-ci mises ensemble à partir de textes et de discours multiples préexistants: d'une part, on retrouve le discours du mal du siècle romantique de Chateaubriand (René, 1802), et de Musset (La confession d'un enfant du siecle, 1836) et d'autre part le scientisme physiognomiste du Médecin de campagne de Balzac (1833) mêlé d'économisme biologique. Bien plus tard ces courants discursifs mèneront (de manière directe ou détournée) jusqu'à la dégénérescence héréditaire des Rougon-Macquart (1871-1893) de Zola, au déracinement tant décrié par Barrès dans Les déracinés (1897), et aux plaisirs fort particuliers du Jardin des supplices (1899) de Mirbeau, en passant par les innombrables études d'hygiène ${ }^{8}$, d'histoire", de criminologie ${ }^{10}$ et de pseudo-science ${ }^{11}$. Le discours sur la dégénérescence, dans toutes ses formes et inflexions - littéraire, scientifique, journalistique, philosophique, etc. - coule comme un vaste fleuve qui traverse le siècle, source intarissable de repré-

8 A. Parrent-1)uchâtelet, De la prostitution dans la ville de Paris considérée sous le rappont de libygiene publique, de la morale et de l'administration, 2 vols. Paris, 1836. Un texte fondamental qui est analysé en termes sociologicuues par Alain Corbin, Les filles de noce: misère sexuelle et prostitution (19" siècle), Paris, Flammatrion, 1982 (1978) et en termes discursifs/littéraires par Charles Bernheimer, Figures of Ill-Repute. Representing Prostitution in NineteenthCentury France, Cambridge, Harvard U. P., 1989.

9 Hippolyte Taine. Les origines de la France contemporaine, Paris, 1875-1894; Max Nordau, La dégénérescence, Paris, 1894 (all. 1892).

10 Cusare Lombroso, Lbonme criminel, Paris, 1887 (it. 1876).

1] Joseph-Arthur de Gobineau, Essat sur l'mégalité des races bumaines, Paris, 1853-55. 
sentations qui servent à des "fins" diverses ${ }^{12}$. Ce discours qui assimile si avidement tant de savoirs diffërents pour fournir la preuve évidente de la dégénérescence rapide de l'Européen(ne), et surtout du/de la Français(e), est quand même à différencier du discours plus limité de la décadence qui, progressivement, se définit dans le dernier quart de siècle ${ }^{13}$. Pourtant, les deux discours font preuve de la même fidélité au modèle socio-biologique (à inflexion socio-darwiniste grandissante) qui domine tout le siècle.

Comme contre-discours esthétique (et idéologique, cela va sans dire) la décadence se distingue par de nombreuses caractéristiques importantes: la marginalité et l'aliénation du héros vis-àvis de la société de masse ${ }^{14}$, le caractère extrême de certaines

12 Une bibliographie des discours de/sur la dégénérescence (pour la période 1789-1914) contiendrait des milliers de titres provenant de tous les discours en circulation à l'intérieur du discours social. C'est en tout premier lieu la quantité importante des textes et des discours qui incorporent de manière obsessionnelle cette thématique au moyen d'un répertoire sẻmiotique commun qui se signale à tout lecteur $\mathrm{du} \mathrm{XIX}^{\mathrm{e}}$ siècle.

13 Avant la fin du xvir ${ }^{e}$ siècle le terme "décadence" signale surtout quelque chose de repréhensible, au sens d'uéchec" ou de "ruine"; les connotations sont surtout d'ordre politique. Dès le milieu du xix $\mathrm{X}^{\mathrm{e}}$ siècle le terme est déjà reconnu au sens moderne de "déviance séduisante". Le mot açuiert la soliclité d'une doctrine, d'une prémisse philosophique, grâce surtout à son emploi par Baudelaire, Gautier, (et à sa manière 'Taine), D'Aurevilly, et Huysmans, entre autres qui l'exploitent comme bannière d'un contre-discours opposé à l'hégémonie réaliste et naturaliste. (A. E. Carter, The Idea of Decadence in French Literature. 1830 - 1900, Toronto, University of 'Toronto Press, 1958, p. 144).

14 Selon l'historien Eugen Weber, "the notion of decadence with which that of the fin de siecle is closely associated was not a burning concern of the vulgar classes, ratber of the literate few. (There was al discrepancy between the concern of the upper classes with decadence (moral, material, social) and the real impnovements in living standards, intellectual equipment, and social opportunities that the popular masses were beginning to perceive, even sometimes to experience." (France. Fin de siècle, Cambridge, Harvard U. P., 1986, p. 13-14). Cette différence de perception basée sur les différences de classe fut sans doute réelle; il y a pourtant peu d'études là-dessus. À titre d'exemple, les personnages principaux des romans décadents (écrits par des bourgeois, notons-le) représentent la décadence soit comme inhérente à la conclition sociale du héros (la noblesse déchue de des Esseintes; ou les origines prolétariennes de Célestine dans Le journal d'une femme de chambre, Mirbeau (1900), et celles de Mouchefrin et Racadot dans Les déracinés); soit comme une condition dont on se délecte (des Esseintes); soit comme imposée par la contamination des classes supérieures (Célestine). Les "origines" et les milieux de la décadence sont des éléments fort entremêlés. 
représentations ${ }^{15}$, l'esthétisation du pathologique ${ }^{16}$, la louange de l'artificiel ${ }^{17}$, le raffinement stylistique ${ }^{18}$, l'emploi des savoirs scientifiques et technologiques à des fins anti-modernistes ${ }^{19}$, entre autres. Si l'intensification obsessionnelle du discours de (et sur) la dégénérescence de la fin de siècle est en grande mesure une expression de l'angoisse (surtout masculine) devant une constellation de phénomènes inhérents au modernisme - la démocratisation de la politique et la montée des classes inférieures, le développement d'une société de masse, l'urbanisation croissante, la sexualité féminine et le fléau des maladies vénériennes, l'envahissement de la technologie dans tous les aspects de la vie quotidienne, et encore bien d'autres -, le discours décadent en constitue le versant esthétisant.

C'est surtout la dimension mythique de la décadence qui devrait attirer notre attention, car du point de vue "objectif " il n'y a aucun fondement empirique pour soutenir les prémisses qui fondent les discours sur la décadence, la dégénérescence, etc. : si, vers 1870 , la supériorité économique de l'Angleterre et de l'Allemagne s'avère incontestable, à la fin du siècle la France atteint à peu près les mêmes conditions de vie que ses voisins ${ }^{20}$. D'une part, la décadence (et la notion plus large de dégénérescence d'où elle sort) est une construction discursive, la représentation

15 On pense par exemple aux supplices du Jardin de Mirbeau, ou à la débauche à la fois intentionnelle et gratuite de la jeunesse entreprise par des Esseintes dans À rebours.

16 Cola s'énonce pour la première fois surtout dans Les fleurs du mal et Le spleen de Paris et revient constamment chez de nombreux écrivains (Verlaine, Huysmans et al.).

17 Le lavement nourrissant de des Esseintes en constitue peut-être le sommet: "L'opération réussit et des Esseintes ne put s'empêcher de s'adresser cle tacites félicitations à propos de cet événement qui couronnait, en quelque sorte, l'existence (qu'il s'était créée; son penchant vers l'artificiel avait maintenant, et sans même qu'il eût voulu, atteint l'exaucement suprême... (p. 332)

18 Huysmans, Villiers de l'Isle-Adam, Corbière, Mallarmé.... cette caractéristique, parfois ouvertement assumée et parfois lancée contre eux comme accusation, revient chez beaucoup d'auteurs: le poème en prose en constitue la meilleure formalisation contre-discursive.

19 Des Esseintes dans $\dot{A}$ rebours et Edison dans L'Ève future en définissent les frontières: foncièrement le discours du savoir scientifique (en médecine et en mécano-cybernétique) sert dans ces cas à rejeter le modernisme, et tout ce qu'il implique.

20. Yves Vadé, "Mythe de la décadence et décadence du mythe", L'esprit de clécadence, Colloque de Nancy, 2. Vol. Paris, Minard, 1980, 1984, II, p. 258. 
124

d'un état d'être, d'une sociopathologie de classe, une réponse à la transformation fondamentale des systèmes symboliques. D'autre part, c'est une reconfiguration épistémologique qui a lieu "sous l'influence conjuguée de la pensée scientifique, de la civilisation industrielle et de nouvelles pratiques sociales" 21 . C'est précisément à cause d'une carence symbolique, d'un "déficit mythique " 22 que le discours de la décadence est appelé à remplir une fonction toute particulière en fin de siècle.

À cette époque déjà certains auteurs s'expriment sur le nouveau rapport entre l'homme et les technologies qui envahissent le quotidien, sur la rapidité du changement, et sur la capacité de l'être humain de s'y adapter. De nombreuses voix énoncent des doutes vis-à-vis de la capacité de l'homme de s'accoutumer au rythme accéléré de la vie. Par exemple, Max Nordau (1892) croit l'homme capable de s'adapter à ce qu'il appelle "la vie frénétique des années 1890 " seulement après de nombreuses générations, peut-être à la fin du $x x^{\mathrm{e}}$ siècle $^{23}$. George Beard (qui a inventé le terme "neurasthénie") croit que la vitesse contribue à une foule de problèmes actuels: la neurasthénie, la dyspepsie nerveuse, les caries, et même la cécité prématurée (!) ${ }^{24}$. Le médecin allemand Willy Hellpach établit un lien entre la pathologie et la vitesse ${ }^{25}$, et même Octave Mirbeau se prononce ainsi dans son "romanreportage" sur l'automobilisme du début du $\mathrm{xx}^{\mathrm{e}}$ siècle:

Il faut bien le dire - et ce n'est pas la moindre de ses curiosités - l'automobilisme est une maladie, une maladie mentale. Et cette maladie s'appelle d'un nom très joli: la vitesse [...] Non pas la vitesse mécanique qui emporte la machine sur les routes, à travers pays et pays, mais la vitesse, en quelque sorte névropathique, qui emporte l'homme à travers toutes ses actions et ses distractions [...] Son cerveau est une piste sans fin où pensées, images, sensations ronflent et roulent, à raison de cent kilomètres à l'heure. [...] Tout autour de lui, et en lui, saute, danse, galope, et est en mouvement, en sens inverse de son propre mouvement. Sensation douloureuse, parfois, mais forte, fantastique et grisante, comme le vertige et la fièvre. ${ }^{26}$

21 Ibial, p. 264.

22 Ibid., p. 261.

23 Citć dans Danicl Pick, Faces of Degeneration. A European Disorder, c. 1848c. 1918, Cambridge, Cambridge University Press, 1989, p. 24.

24 Anerican Neriousness: Its Causes and Conseguences, New York, 1881, p. 116.

25 Nervosität und Kultur. 1902.

26 La 628-ES, Paris, Chatpentier, $1917|1907|$, p. 6-7. 
Comme nous le verrons, la notion de rythme accéléré de la vie, de vitesse, qui s'énonce en termes d'une incapacité d'adaptation, se transforme au $\mathrm{xx}^{\mathrm{e}}$ siècle: les personnages de Crash établissent un tout autre rapport à ce phénomène en s'y adonnant complètement. Le discours décadent d'avant les années 1860 relève surtout des discours littéraires, empruntés largement au romantisme: c'est le genre de "décadentisme" littéraire qui se retrouve chez Gautier et Baudelaire. Pourtant, après cette période c'est surtout le discours scientifique qui commence à se faire sentir beaucoup plus dans les écrits "décadents". En tout premier lieu, le discours médical - qui se distingue par ses innombrables études sur la névrose, l'épilepsie, la catalepsie, l'hystérie, le somnambulisme, etc. - sert de source inépuisable pour l'épanouissement continuel du discours sur la dégénérescence en général ${ }^{27}$. À témoin ce compte-rendu de Zola sur Germinie Lacerteux:

Il y a...une relation intime entre l'homme moderne, tel que l'a fait une civilisation avancée, et ce roman du ruisseau, aux senteurs âcres et fortes. Cette littérature est un des produits de notre société qu'un éréthisme nerveux secoue sans cesse. Nous sommes malades de progrès, d'industrie, de science; nous vivons dans la fièvre, et nous nous plaisons à fouiller les plaies, à clescendre toujours plus bas, avides de connaitre le cadavre du coeur humain. ${ }^{28}$

Quand l'éphémère revue Le Décadent parut en 1886, le rédacteur en chef, Anatole Baju, annonça que les décadents comme type furent "les précurseurs de la société de l'avenir" ${ }^{29}$. À un rythme accéléré et dans un nouveau rapport avec la vitesse et la technologie, les personnages du roman Crash vivent cet avenir.

27 Dans son compte rendu sur Madame Bovary (publié dans L'Aitiste du 18 octobre 1857), 13audelaire voit clairement les liens discursifs entre le discours littérare et le discours médical: "Lhystérie! Pourquoi ce mystère physiologicue ne ferait-il pas le fond et le tuf d'une cuvre littéraire, ce mystère que l'Acádémie de la médecine n'a pas encore résolu, et qui, s'exprimant dans les femmes par la sensation d'une boule ascendante et asphyxiante (je ne parke cue clu symptôme principal), se traduit chez les hommes nerveux par toutes les impuissances et aussi par l'aptitude à tous les excès?" Giuvres complètes, Paris, Gallimard, coll. "Bibliothèque de la Pléiade", 1961, p. 654.

28 Émile Lola, "Germinie Lacerteux", Mes Haines, Guvres completes, tome 10. Paris, Cercle du livre-précieux, 1968, (1866), p. 71.

29) Cite dans Richard Gilman, Decadence. The Strange Life of an Epithet, New York, Farrar, Strauss and Giroux, 1979, p. 76. 


\section{La « nouvelle décadence " dans Crash ${ }^{30}$}

Si la "décadence" du dernier quart du $\mathrm{xIX}^{\mathrm{e}}$. siècle constitue une réponse, un contre-discours, à un phénomène socio-historique précis - la transition au modernisme - ce que j'appelle ici la "nouvelle décadence" qui informe le roman Crash (et encore d'autres produits culturels de l'époque) en constitue à la fois un prolongement et une ré-écriture dans un contexte "fin de siècle" renouvelé. Certains disent que les fins de siècles se ressemblent: que ce soit vrai ou non, "elles ont certainement en commun la conscience d'être une fin " ${ }^{31}$. Dans Crash l'auteur nous offre un monde où le désir (pour se réaliser) est fort technologisé, où la sexualité devient le seul moyen d'expression personnelle, la seule "langue" de transcendance, et où l'homogénéisation banale du quotidien n'est vivable qu'au moyen de l'expérience fétichisée, en l'occurrence celle de l'accident de voiture. Dans ce texte l'auteur pousse à l'extrême (comme l'ont fait ses prédécesseurs au siècle précédent) un certain nombre d'éléments thématiques, épistémologiques, et discursifs pour analyser un monde où l'interpénétration du technologique et de l'humain devient pathologique ${ }^{32}$. Les personnages de $C r a s h$ inscrivent l'histoire de la vitesse, comme force médiatrice et "créatrice" dans leurs vies, sur la réalité ultime de leurs corps. La connaissance de l'autre, de la sensation, et de l'identité se fait ici au moyen de la technologie d'automobile et de la "reconstruction" du corps déformé, ou à déformer. À la fois victimes et maîtres, les personnages de ce monde essaient de façonner et de connaître une réalité qui n'appartient qu'à eux. Les tatouages fièrement affichés et les boucles d'oreilles savamment attachées ici et là aux corps des jeunes (et parfois des moins jeunes) de nos jours constituent une esthétique en quelque sorte "incorporée" 33 . De manière sem-

30 Dans ce qui suit toutes les références à Crash sont indiquées entre guillemets dans le texte.

31 Pierre Brunel, "La légende des fins de siècle", Cabiers de l'Herne, Huysmans, Paris, Éditions de l'Herne, 1985, p. 13.

32 Cette rêflexion est poursuivie dans un texte en voie de préparation, "Variations on a Theme: The New Decadence in Crash, Kissed, sbopping and Trainspotting".

33 Il est fort significatif que David Cronenberg a ajouté au film une scène de tatouage qui n'existe pas dans le roman et ainsi souligne le processus d'inscription corporelle littérale et symbolique à l'œuvre dans le texte. Vers la fin du scénario Vaughan et Ballard se retrouvent dans un salon de tatouage: 
blable, les blessures que portent les personnages de Ballard sont autant de tentatives de ré-écriture de leur identité corporelle et psychique unique.

\section{La vitesse comme moyen de transformation}

"Réalisme magique: cette formule est sans doute la meilleure définition que l'on puisse donner de l'esthétique de la vitesse. "34 Telle est l'appréciation de Claude Pichois du mode représentationnel où figure la vitesse depuis plus de cent ans. Pour sa part, David Pringle nous propose que dans le cas de James Ballard, "Ballard's strengths are not those of a realistic social novelist [...] he is a symbolic fantasist, and bis characters are usually personifications of psychological urges rather than "real people" "35. La représentation de la modernité en terme de vitesse se fait depuis longtemps. Un élément dans la mythologie moderniste veut que l'homme moderne détienne dans ses mains (tel le démiurge) les outils qui lui permettent de dépasser toutes les expériences banales et contingentes afin de se transformer, spirituellement et physiquement. Au niveau discursif, la transformation la plus fondamentale qui se fait dans Crash est celle de la ré-écriture du discours du désir par le discours de la consommation (de l'autre, voire de soi-même) au moyen de la vitesse. À la fois l'outil dont se sert l'homme pour contrôler le temps et l'espace et la métaphore principale pour le monde artificiel qu'il s'est créé, la voiture exige qu'on s'adapte à elle. La voiture transforme notre rapport au temps et à l'espace, ainsi que notre environnement physique, et en plus constitue un instrument puissant de destruction; mais ici, comme chez les Futuristes, l'instrument de destruction se trans-

"james enters to discover Vaugban getting a wound tattoo on bis abdomen, one that looks as though it could bave been made by the fluted lower edge of a plastic steering wheel. [...]. We close on the fresh tattoo on James's inner thigh. It looks more like a cartoon version of a wound than a real wound." (Cronenberg, op. cit., p. 54-55) On remarque que la blessure de Ballard resscimble à un "cartoon version" comparé à celle de Vaughan: l'hyper-réalisme l'emporte sur la représentation réaliste, ce qui est en accord avec le mode représentationnel dominant du roman.

34 Claude Pichois, Littérature et progrès. Vitesse et vision du monde, Neuchâtel, Éditions de la Baconnière, 1973, p. 123.

35 David Pringle, Earth is the Alien Planet. J. G. Ballard's Four Dimensional Nightmare, San Bernardino, CA, Borgo Press, 1979, p. 51. 
forme à rebours en instrument de création à travers la destruction ${ }^{36}$.

L'exaltation de la vitesse grâce à la machine se trouve au centre du culte moderne de l'artificiel en général, mais elle semble distinguer le monde "fin de siècle" de Huysmans de celui de Ballard: là où chez Huysmans, au lieu de voyager, des Esseintes s'entoure dans son petit monde à Fontenay de poissons mécaniques et de tout ce qui peut ajouter à lillusion d'avoir voyagé, dans Crasb la vitesse et l'ambiance de la voiture deviennent ensemble le catalyseur nécessaire à l'éclosion du désir. Cet environnement hautement technicisé mais en même temps naturalisé par rapport au corps humain est le Nouveau Monde de l'expérience authentique.

Le rapport entre l'espace et le temps est un des rapports fondamentaux que le modernisme a déconstruit, du moins dans sa forme traditionnelle. Les travaux des savants ainsi que les mises en place de la technologie dans la vie quotidienne ont mené à la reformulation de ce rapport aux plans théorique et abstrait tout comme au plan pratique ${ }^{37}$.

Dans Crash, il y a un rapport "harmonique" qui s'établit entre les corps et le mouvement physique. Ballard commente à maintes reprises la manière dont Vaughan consomme la vitesse et est consommé par elle: "Dans ce royaume illuminé par la violence et la technologie, lancé à 160 à l'heure sur une autoroute vide, il roulait éternellement, dépassant les stations-service désertes le long de vastes plaines, guettant l'apparition face à lui d'une unique voiture" (p. 16). Vaughan manipule les corps de ses partenaires sexuels (en l'occurrence ceux des prostituées) en rapport avec le monde qui l'entoure dans la voiture et le mouvement de celui-ci: "Prenant appui sur son coude, il a repris son va-et-vient dans la main de la fille. Il semblait participer à une danse rituelle, aux figures strictement réglées, qui aurait pour objet de célébrer l'électronique et le design, la vitesse et la ligne d'une auto futuriste" (p. 160); "C'est lorsque nous avons atteint le toboggan de

36 Voir le chapitre 5 sur "Speed" dans Stephen Kern, The Culture of Time and Space: 1880-1918, Cambridge, Harvard U. P., 1983, p. 109-130.

37 Deux études qui essaient de saisir les conséquences de cette reformulation des rapports dans des perspectives différentes sont: Wolfgang Schivelbusch, The Railuay Joumey. The Industrialization of Time and Space in the 19th Century, New York, Berg, 1986, et Stephen Kern, op. cit. 
l'aéroport que cette union du sexe et de la technologie a atteint son plus haut point d'intensité "(p. 160). Quand Catherine et Vaughan font l'amour sur le siège-arrière au lave-auto, James les observe et analyse ainsi leur comportement: "Leur étreinte m'apparaissait comme un rituel vidé de contenu sexuel, le débat formel de deux corps exposant leur conception du mouvement et du choc" (p. 181); "Il [Vaughan] ordonnait son corps selon une suite de positions, déchiffrait minutieusement le code de ses membres et de ses muscles"; ils sont "Deux humains semi-métalliques surgis d'un avenir lointain faisa[nt] l'amour sous une charmille chromée" (p. 182). Ballard est conscient de la manière dont les corps répondent à la vitesse - * Je me suis rendu compte que je pouvais pratiquement contrôler le coït qui se déroulait derrière moi par ma façon de conduire" (p. 162). Cette correspondance quasi baudelairienne décrit une sorte de communication entre deux domaines différents jusqu'au point où l'animé et l'inanimé se mélangent de manière presque indistincte, comme pour l'odeur corporelle de Vaughan: "Un certain relent d'huile à moteur et de sécrétions rectales montait de son corps" (148).

La vitesse se matérialise surtout comme langage dans les inscriptions sur les corps des personnages. Évidemment, Gabrielle est l'exemple le plus frappant de la transformation corporelle effectuée par la vitesse. Ballard comprend bien ce qui lui est arrivé : "Cette aimable jeune femme, avec ses aimables rêveries érotiques, était née une seconde fois dans les lignes brisées de sa voiture de sport broyée" (p. 115). Il voit l'histoire de la vitesse inscrite sur son corps: "La carrosserie broyée de la voiture de sport l'avait changée en une créature à la sexualité libre et perverse. Dans le tablier froissé et la fuite du fluide refroidissement, toutes les déviations latentes de sa sexualité aient été libérées. Ses cuisses atrophiées et ses mollets affaiblis étaient les modèles de perversions fascinantes" (p. 115). Du point de vue sémiotique, ce sont "les éléments d'une anthologie pratique de la dépravation, la clé d'une sexualité différente" (p. 115), celle-ci se réalisant par la plaie dans sa cuisse qui remplace l'organe sexuel "usuel" quand Ballard lui fait l'amour: "Cet orifice pervers, véritable invagination d'un organe sexuel encore embryonnaire, m'a remis en mémoire mes propres blessures, les petites cicatrices qui portaient encore l'empreinte du tableau de bord et des commandes" (p. 198). Même les mannequins du Road Research Laboratory portent les inscriptions de la destruction à venir par l'accélération de la 
130

voiture, "Leurs visages étaient peints de signes ésotériques" (p. 141). Le processus de transformation aboutit à l'accident de Seagrave qui meurt in drag pour ainsi dire dans sa tentative de contrefaire la mort des vedettes d'Hollywood: "Le visage amaigri et épuisé de Seagrave était recouvert d'une croûte de verre de sécurité. On eût dit que son cadavre était en train de cristalliser, quittant enfin cette prison dimensionnelle étriquée pour un univers plus beau" (p. 206). L'expérience transformatrice arrive ici à son ultime réalisation.

De nombreux autres exemples se présentent dans le texte. Quand Helen Remington quitte sa voiture après l'accident avec Ballard, "Ses brusques mouvements de tête et sa démarche peu sûre paraissaient imiter les lignes tordues de nos carrosseries" (p. 30). Â l'hôpital après l'accident, Ballard rêve de corps sur lesquels sont inscrits les signes de la vitesse après des accidents imaginés: "Un accident au décollage de Londres et son bas-ventre serait marqué pour des années par la boucle de la ceinture de sécurité qui lui écraserait les chairs" (p. 36), et il lit sur son propre corps l'empreinte du monde technicisé: "Je me suis dit en contemplant ces ecchymoses qu'un spécialiste aurait pu deviner le modèle et l'année de ma voiture à partir du réseau formé par mes blessures" (p. 37). Plus tard, Ballard examine sa voiture dans la "police pound" et lit son avenir dans le cadavre "exquis" de sa voiture: "Alors que je contemplais ce petit musée de la provocation et du possible, l'intérieur défoncé de la voiture, pareil à quelque bizarre machine modifiée à l'usage d'un grand invalide, m'est apparu comme un modèle idéal pour tous les futurs accélérés de mon existence" (p. 83; je souligne). À partir de ce premier accident, c'est une ouverture qui se présente à lui. Et puisque cette voiture est hors service il en loue une autre et éprouve de nouvelles sensations en la conduisant:

Roulant le long de Western Avenue, j'envisageais ma mort éventuelle parmi toutes ces fictions accumulées; la découverte de mon corps mêlé aux images d'une centaine de séries policières et de mélodrames oubliés qui inscriraient, longtemps après qu'un changement des grilles de programmes les aurait envoyés dormir au fond d'un tiroir, la dernière ligne de leur générique final sur ma peau. (p. 73)

Chaque voiture devient une "fiction", une narration en mouvement qui laisse son empreinte. Les hiéroglyphes de cette nouvelle "religion" de la vitesse sont bien lisibles, mais seulement par les 
initiés. À une nouvelle gamme d'expérience, il faut un nouveau langage.

\section{L'économie du désir}

Il y a une économie du désir qui est à l'ouvre dans le texte. Tout comme la vitesse, Vaughan remplit le rôle actantiel de catalyseur: c'est lui qui médiatise l'intimité des personnages principaux et permet le fonctionnement des échanges symboliques à travers la sexualité. Sans lui et sa poursuite de la sexualité morbide il n'y a pas de communication entre eux. Au lit, Catherine et James éprouvent leurs moments les plus intenses ensemble au moyen de Vaughan, car ils se racontent la manière de faire l'amour avec lui (p. 131-134). Comme tout catalyseur, Vaughan accélère l'établissement des nouveaux rapports entre les éléments en question, qu'il s'agisse des rapports entre les êtres humains, ou ceux qui se font entre les hommes et la technologie. Ayant accompli sa tâche, il est libre de mourir à la fin de la narration car sa présence n'est plus nécessaire aux rapports entre Catherine et James, ni à ceux entre Helen et Gabrielle ${ }^{38}$. Le catalyseur joue normalement un rôle très précis mais quand même limité dans tout processus d'interaction. Au niveau actantiel, Vaughan est remplacé de façon permanente par la vitesse.

Si d'une part Vaughan peuple ses fantasmes d'êtres marginalisés ("excited scbizophrenics", "manic-depressives", "luckless paranoids", "sadistic charge nurses", "lesbian supermarket manageresses", "autistic cbildren", "mental defectives", ou "a mentally defective girl"), ou de vedettes d'Hollywood (une sorte de marginalisation invertie par le système de consommation), les personnages du roman eux-mêmes sont tous "bien rangés" du point de vue de leurs origines sociales et de leurs emplois. Pourtant, tous partagent ce que James reconnaît chez Catherine comme "une saine curiosité pour tout ce qui possédait un caractère de perversité" (p. 62). Les nouvelles configurations dues aux effets de la vitesse - "Ces rencontres de sexes déchirés et de sections de caisse ou de planche de bord formaient de troublants modules, les unités monétaires d'une circulation nouvelle de la douleur et

38 "Interview with Conenberg". URL: http//:www.fff.com/crash/cmp/cronenberg-interview.html\#people (7 mars 1997). 
du clésir" (p. 151) - établissent un nouveau système d'échange symbolique. L'expansion de ce système se voit dans la série incessante d'accouplements hétérosexuels et homosexuels où la différence sexuelle elle-même devient quasiment insignifiante. Ce qui compte, c'est le moment même de l'échange: "L'accident était la seule expérience réelle que j'eusse connue depuis des années" (p. 49) dit James après avoir éprouvé le premier accident et, bien plus tard, après avoir fait l'amour avec Vaughan, il observe: "Un sentiment de paix profonde m'a envahi, fait en partie de mon amour pour Vaughan, et en partie de la tendresse que je ressentais pour la charmille métallique qui nous abritait. (p. 224).

Le texte est comme vide d'émotions: il y a des sensations fortes, "le mystérieux érotisme des blessures" (p. 30), mais peu de représentations d'états émotionnels chez ces personnages. C'est plutôt la volonté, le désir, l'obsession qui dominent chez les personnages ét leurs pratiques constituent le système symbolique qui remplace celui des émotions. Puisqu'on doit inventer une nouvelle façon d'établir et de maintenir le contact humain, le catalyseur de la vitesse reste toujours nécessaire dans cette économie. Ainsi dans la dernière scène du livre, après la mort de Vaughan, Ballard pense à ce que sera l'avenir: "Déjà, je le savais, j'étais en train de réunir les éléments de ma propre mort automobile" (p. 250) et le roman se termine par une image hyper-réelle: "Cependant, le flot incessant de la circulation progressse sur le toboggan. Les avions quittent les pistes de l'aéroport. Ils emportent ce qui survit de la semence de Vaughan vers les tableaux de bord et les calandres de milliers de voitures à l'instant qu'elles s'écrasent, vers les jambes déjetées de millions de passagers" ( $\mathrm{p}$. 250). La nouvelle économie du désir en est venue à s'exprimer dans un langage nouveau, mais le catalyseur du mouvement, de la vitesse reste nécessaire.

\section{L'obsession, le fétiche, le rituel et l'artificiel}

Crash est un texte obsessionnel, voire pathologique, de tous les points de vue: la thématique se limite à une gamme étroite de problèmes; les personnages sont peu nombreux et tous motivés par des désirs obsessionnels; le style même reprend l'obsession au moyen d'insistantes répétitions de lexique, d'images et d'éléments narratifs. Dans la nouvelle économie du désir, le mode dominant est celui de fétichisme. Comme Sisyphe, ces personna- 
ges sont condamnés à un rituel rythmé (donc à une certaine "vitesse") qui ne trouve sa fin que dans la mort ${ }^{39}$. Le fétichisme qui remplit leurs vies remplace toute croyance politique, religieuse, etc. (ou plutôt, l'exprime de manière transformée, déplacée) et Vaughan devient en quelque sorte leur Messie, le catalyseur de leur éclosion spirituelle ${ }^{10}$. La sexualité remplace toute communication entre les personnages de l'histoire: ce n'est qu'à l'intérieur de la sexualité ritualisée, de ce langage particulier qui ne s'articule le plus souvent que dans un milieu (réel ou suggéré) d'automobile, que les personnages arrivent à entrer en contact, physiquement et spirituellement, les uns avec les autres. Ballard et Catherine, Ballard et Gabrielle, Ballard et Vaughan, Ballard et Helen, Vaughan et les prostituées, etc. : la liste des permutations et des combinaisons pourrait continuer. Système sémiotique, la communication sexuelle qui se présente ici contient de nombreuses possibilités, variantes, etc. qui sont toutes à la poursuite du référent absolu (et inexistant) de la transcendance. Le langage utilisé par le narrateur pour décrire la sexualité est clinique et vise la précision d'un langage artificiel/scientifique, tout comme les inscriptions de la vitesse sur le corps de Vaughan: "Ensemble, ils composaient un langage précis de sensation et de soulfrance, d'érotisme et de désir" (p. 106). Le nouveau rituel en vient à constituer l'essentiel des rapports humains et remplace les rituels usés.

L'artificiel technologique lie les individus: non seulement les voitures, mais d'autres technologies également: "Nos sentiments cristallisaient autour des objets neufs de la technologien (p. 41), dit Ballard à propos du téléphone, et une autre technologie lui rappelle la sexualité :

Au moment où je clémarrais, son appareil photo est venu atterrir à côté de moi sur le siège. Invisibles, des souvenirs de souffrance et d'exitation scintillaient dans l'obscurité de la pellicule

39) Dans le film, Cronenberg accentue le mouvement ritualisé, le rythme lent qui fonctionne comme une sorte de pendant à la notion de vitesse comme mouvement frénétique.

40) Je ne poursuis pas dans ce contexte la dimension "religieuse" du roman. Poutant, la mortification de la chair; le rôle quasi messianique de Vaughan; l'unification de la chair et du métal qui rappelle toute une série de notions cle transcendance et de consubstantiation; les images de résurrection, et encore d'autres éléments méritent d'être analysés par rapport au ciscours religicux. 
134

qu'ils avaient impressionnée. Derrière moi, les muqueuses les plus sensibles de Catherine, elles aussi, sécrétaient doucement leur troublante chimie. (p. 178)

L'artificiel et le naturel sont mis sur le même plan. L'obsession de Vaughan et de Seagrave pour les vedettes d'Hollywood, comme l'obsession généralisée pour les voitures, nous situent dans un monde de constructions artificielles qui dominent le roman.

Il y a donc un déplacement à l'infini, une mise en scène de la différance. Le comportement des personnages, dans sa ritualisation, est un comportement primitif, de tribu - un comportement qui finalement n'est pas trop éloigné de celui de des Esseintes. Et encore, comme des Esseintes qui se coupe du monde ("Est-ce qu'il ne s'était pas mis lui-même au ban de la société? "11 se ditil), les gens dans ce petit groupe autour de Vaughan s'adonnent à leur quête.

La dimension rituelle de l'obsession a un effet sur la vitesse. On fait l'amour surtout dans la voiture, espace rituel médiatisé. Parfois, la voiture roule, parfois non. Mais le rituel a son propre temps interne, qui est quand même comme synchronisé avec celui de la voiture. Le narrateur parle de "gestes codés" (p. 31), de "ballet rituel" (p. 30), et en parlant des personnages dit que "leurs moindres mouvements semblaient réglés d'avance" (p. 31). À la fin du roman Ballard fait l'amour avec Vaughan dans "les formes stylisées de l'habitacle" (p. 223) pendant qu'il regarde "les centaines de gondoles radieuses qui glissaient sur l'autoroute audessus de nos têtes" (p. 223).

Le culte de l'artificiel démontre la supériorité de l'homme visà-vis de la nature, le refus de toute notion romantique et constitue la caractéristique fondamentale de la décadence au sens dont le $X I X^{C}$ siècle entend le mot. Dans les innombrables variantes des accidents et des positions sexuelles l'esprit du Marquis de Sade semble également hanter le roman: "Vaughan construisait d'infinies variations à partir de ces accidents.... (p. 22).

Le monde que voit Ballard de son appartement est circonscrit par les autoroutes, un monde artificiel construit pour canaliser la vitesse contrôlée: 
En examinant attentivement l'étendue silencieuse, je me suis rendu compte que tout le paysage qui formait le cadre de mon existence était maintenant délimité par un horizon entièrement artificiel. Les voies surélevées, les garde-corps et les accotements de l'autoroute, les bretelles d'accès, les échangeurs enserraient les véhicules comme les parois d'un cratère de plusieurs kilomètres de diamètre (p. 65).

L'artificiel domine le roman à tous points de vue et les personnages l'assument pleinement dans leur tentative de se redéfinir comme êtres humains.

\section{Le laboratoire}

La notion de laboratoire est un élément central de la narration: que ce soit comme métaphore, espace physique, thématique ou élément discursif, elle revient constamment. Tout comme des Esseintes dans $\grave{A}$ rebours, les personnages dans ce roman sont engagés dans une sorte d'expérience (sinon scientifique du moins une expérience qui possède sa propre logique): l'espace de l'expérience est encore une fois le corps humain, sauf que cette fois cet espace connật une extension technologique grâce à la voiture. Afin de préparer sa grande expérience (l'union finale avec la vedette, Elizabeth Taylor), le Dr. Robert Vaughan, autrefois spécialiste en informatique et chez qui Ballard reconnait maintenant "le savant comme blouson noir" (p. 77), collectionne toutes sortes d'informations sur les accidents pour les "analyser" dans son laboratoire chez Seagrave. Il se documente minutieusement en entassant photographies, livres, et réponses aux questionnaires qu'il distribue: "J'ai fait quelques sondages en vue du programme. Jette un coup d'œil sur ces questionnaires, et dis-moi si je n'ai rien oublié " (p. 147), dit-il à James. Celuici ressent fortement toute cette dimension "scientifique":

Sa présence avait affecté ma façon de conduire. Je soupçonnais qu'en réalité je me préparais pour un deuxième accident, mais certe fois sous les yeux de Vaughan. Même les avions géants qui prenaient leur vol sur les pistes de l'aérodrome m'apparaissaient comme des combinaisons de sensations fortes et d'érotisme, de clésir et de châtiment, des figures toutes prêtes à s'imprimer dans ma chair. Les énormes bouchons qui obstruaient l'autoroute rendaient l'atmosphère étouffante. J'en venais presque à croire que c'était Vaughan lui-même qui avait attiré tous ces véhicules sur l'asphaite harassé, en vue de quelque test psychologique sophistiqué (p. 165-166). 
136

On se souviendra que dans la voiture, avec les prostituées, Vaughan manipule littéralement les corps des femmes "comme s'il réglait le bouton de quelque bizarre appareil dans un laboratoire" (p. 159). De manière semblable, il manipule Seagrave pour réaliser ses expériences d'accidents de vedettes: “Pour une raison mal définie, elle [Vera Seagrave] dégageait Vaughan de toute responsabilité bien que celui-ci - je l'ai compris plus tard, mais elle devait déjà le savoir - utilisât son mari comme sujet d'expérience" (p. 108). Le plus souvent, Vaughan agit en observateur, à part les moments où il devient lui-même un élément de l'expérience. Mais il dépasse ce rôle lorsqu'il écrase le piéton.

La notion de laboratoire s'étend à d'autres personnages. Après l'accident avec James, Helen Remington quitte son emploi à l'aéroport pour travailler dans un laboratoire, le "Road Research Laboratory" dont le but même est de créer, mesurer et enregistrer des accidents. Vaughan, avec les autres, observe l'expérience plusieur's fois, même au ralenti, grâce aux appareils, et il s'en trouve heureux: "Loin du centre d'essais, il a retrouvé toute son agressivité. Les voitures qui passaient semblaient aiguiser son appétit" (p. 146). Quand Ballard fait l'amour avec Gabrielle, il vit l'événement également en termes d' "expériences": "Nos coïts étaient des expéditions éprouvantes ${ }^{42}$ (p. 197).

Le laboratoire renvoie également au langage. Catherine a besoin de se remplir l'esprit de représentations fantaisistes d'une autre femme quand elle fait l'amour avec James, qui en est fort conscient: "Ces descriptions me faisaient penser à un langage en quête d'objets, ou même à l'annonce d'une sexulalité nouvelle, séparée de toute expression physique" (p. 44). Soit que les personnages parlent entre eux, soit que le narrateur nous raconte l'histoire, il y a un effet de détachement et d'aliénation dans le langage car on parle de la sexualité, des corps, et des actes de manière quasiment clinique. Encore une fois, on revient à l'artificiel.

\section{$* *$}

Si les rapports entre l'ancienne et la nouvelle décadence sont aussi évidents que je le crois, et si ceux entre le psychopathologi- 
que et le sociopathologique restent aussi étroits à la fin de notre propre siècle qu'à la fin du siècle précédent, c'est que les sociétés humaines n'en sont qu'au début des nouvelles relations qui s'établissent entre l'être humain et l'environnement technicisé. L'importance accordée ici à la notion de vitesse sous toutes ses formes relève à la fois de la réalité empirique de notre existence quotidienne, des changements dans notre appareillage épistémologique, et des systèmes symboliques dont nous nous servons pour faire sens dans notre monde en mouvement. Les représentations à la fois fascinantes et troublantes de Crash témoignent du pouvoir du discours poétique de répondre à notre nouvel environnement social. 